\title{
Making fish a little more human: a zebrafish hematopoietic xenotransplant model is improved by the expression of human cytokines
}

\author{
Owen J. Tamplin \\ Department of Cell \& Regenerative Biology, University of Wisconsin-Madison, Madison, WI, USA \\ E-mail: OWEN J. TAMPLIN - tamplin@wisc.edu
}

doi:10.3324/haematol.2020.256909

$\mathrm{F}$ or decades the mouse xenotransplant model has allowed us to track the behavior of transplanted human cells. Whether it is transplantation of hematopoietic stem and progenitor cells (HSPC) or leukemic cells, immunodeficient mice provide a means of measuring homing, engraftment, and stem cell dynamics. ${ }^{1-3}$ However, there are limitations to these mouse models that have made it difficult to advance certain aspects of HSPC and leukemia research. For example, immunodeficient mouse models such as NSG (NOD-scid IL2R $\left.\gamma^{\text {null }}\right)^{4}$ are expensive to maintain, making them prohibitive to scaling for screening purposes. Direct intravital imaging can be performed on mouse transplant models, ${ }^{5,6}$ but because of its technical challenges, this technique is not suitable for use in a large number of animals. To tackle the diversity of human HSPC and leukemias, there is a need for a model that can be housed at high density, easily treated with therapeutics, and directly imaged if necessary. The zebrafish has become well-established as an alternate model that can satisfy these additional research needs.

Although there are immunodeficient adult zebrafish models available, ${ }^{8}$ working with embryos [0-3 days post-fertilization (dpf)] and larvae (3-30 dpf) has its own advantages. The zebrafish does not fully develop adaptive immunity until juvenile stage (>30 dpf), making it ideal as a xenotransplant model. Also, the smaller and more transparent embryos are particularly amenable to live imaging. As one adult female can spawn up to 200 embryos per week, and larvae can be maintained at high density, it is a truly scalable model. It is easy to deliver cells into the circulation by microinjection. If injected cells are fluorescent, made so by either genetic modification or dye label, they can be immediately tracked from the injection site as they home to and interact with the niche. One caveat of this model is that the ideal temperature for maintenance of zebrafish is $28.5^{\circ} \mathrm{C}$, so a compromise temperature of $35^{\circ} \mathrm{C}$ must be used in xenotransplants, ${ }^{9}$ allowing mammalian cells to be compatible with the host environment. Even with these advantages, there has still been room for optimization of the zebrafish as a recipient for human HSPC and leukemic cells.

The hematopoietic ontogeny, genetic programs, and cell lineages are highly conserved among vertebrates, making results from zebrafish translate exceptionally well to humans. ${ }^{10,11}$ However, the cytokines that are essential as regulators of hematopoiesis are not well conserved. ${ }^{12}$ This led researchers to "humanize" mouse models by introducing human cytokines, either by injection or transgenesis. ${ }^{13,14}$ Following this approach, but with the added advantages of the zebrafish model, Berman's group developed a humanized zebrafish that expresses factors critical for support of human HSPC and leukemia cells. ${ }^{15}$ To develop this model, they selected the human cytokines GM-CSF, $\underline{\text { SCF} / K I T L G, ~ a n d ~ S D F 1-~} \alpha / C X C L 12$ (named "GSS") as top candidates to support human cells in the zebrafish model. They expressed these factors in vivo by gen- erating a triple transgenic zebrafish line to support transplanted human hematopoietic cells. Human CXCL12 is driven by the zebrafish $\mathrm{cxcl}_{12}$ promoter, and GM-CSF and SCF were doxycycline inducible. Strikingly, expression of all three cytokines had the effect of promoting human cell survival and differentiation.

Previous work has been done in Berman's laboratory to develop zebrafish leukemia xenotransplant models., ${ }^{9,16}$ Other groups have successfully transplanted adult human CD34 ${ }^{+}$ $\mathrm{HSPC}^{17}$ and mouse HSPC ${ }^{18}$ into zebrafish larvae. Interestingly, these human cells were found to trigger similar cellular behaviors in the niche as were seen during endogenous zebrafish HSPC lodgement, ${ }^{19}$ highlighting the similarity between mammalian and zebrafish HSPC.

To evaluate their new GSS model over previously developed models, they applied a number of metrics to test its function: (i) migration; (ii) proliferation; (iii) chemotherapy response; (iv) clonality; and (v) host survival. Ultimately, their goal was to make a better microenvironment for human HSPC and leukemic cells. First, to test migration, they injected Jurkat human T-acute lymphoblastic leukemia cells into the yolk of single $\underline{S} D F 1-\alpha / C X C L 12-o v e r e x p r e s s i n g ~ l a r v a e ~(S$ fish) because this cell line expresses high levels of CXCR4. There was little initial response and they reasoned that the injection site was not optimal (yolk and not circulation), and SDF1- $\alpha / C X C L 12$ expression levels of the transgenics were low. To compensate they irradiated recipient larvae and were able to induce a migratory response of transplanted cells in SDF1- $\alpha / C X C L 12-$ expressing transgenics.

Next, to test proliferation, they transplanted a human Down syndrome acute myeloid leukemia (AML) cell line (CMK) into transgenic larvae carrying the doxycycline-inducible GMCSF/CSF2 and SCF/KITLG transgenes (GS fish). Expression of these human factors in the zebrafish larvae proved effective, as CMK cells were more proliferative 3 days post-injection (dpi). However, as in the experiment above, xenotransplanted cells were injected into the yolk, which can have the effect of trapping the cells and reducing their access to circulation. Following these results, the authors proceeded to perform all injections directly into the circulation.

The authors then combined transgenics to establish the triple transgenic GM-CSF, SCF/KITLG, and SDF1- $\alpha / C X C L 12$ (GSS) line. As proof-of-concept for drug screening, the authors first chose the chemotherapy medication cytarabine. The CMK cell line they used was derived from a 10-year old Down syndrome patient with AML who was responsive to this drug. Zebrafish embryos and larvae are well-suited for mediumthroughput drug screening because the entire organism can be soaked in drug. These treatments produce an in vivo phenotypic read-out of drug effects. Interestingly, the drug was only effective on xenografts in the GSS line but not in control fish. This response was not fully explained in the study; however, the increased proliferation of leukemic cells from GSS 
cytokines may have made their reduction by chemotherapy treatment more obvious. Alternatively, the GSS cytokines may directly affect the CMK cells, allowing them to have a more physiological response to the drug.

Following the promising treatment of CMK xenograft larvae with cytarabine, the authors injected four different primary AML cells into the triple GSS fish at $3 \mathrm{dpf}$. Consistently, AML cells in GSS fish reduced survival of the host, presumably because cells were able proliferate more in the presence of human cytokines. Diminished survival at 3 dpi was not simply because of the xenotransplant procedure, as introduction of human HSPC into the circulation of zebrafish larvae did not decrease survival. Another measure of these xenografts was clonality, which was determined by error-corrected RNA sequencing. Twice as many individual clones from heterogenous human AML were preserved in the GSS model compared to controls.

Together, the above experiments demonstrated that expression of human pro-hematopoietic cytokines in the GSS transgenic model provided a superior microenvironment for xenografts of human HSPC and leukemic cells. This was assessed by increased human cell migration with human CXCL12, increased proliferation with GMCSF/CSF2 and SCF/KITLG, decreased survival of the recipient, better response to chemotherapy, and increased clonality of xenotransplant cells. Despite the many advantages of the zebrafish, there are still some limitations. For example, xenograft human cells only survive for a few days at most. This prevents the long-term tracking of human cell engraftment and disease progression in the host that is possible in mammalian transplant models, such as the mouse ${ }^{1-3}$ or non-human primate. ${ }^{20}$ Overall this novel xenotransplant model has many potential applications, from live imaging of leukemia interaction with the niche, to the rapid optimization of patient-specific chemotherapy.

\section{References}

1. Bonnet D, Dick JE. Human acute myeloid leukemia is organized as a hierarchy that originates from a primitive hematopoietic cell. Nat Med. 1997;3(7):730-737.

2. Lapidot T, Pflumio F, Doedens M, Murdoch B, Williams DE, Dick JE. Cytokine stimulation of multilineage hematopoiesis from immature human cells engrafted in SCID mice. Science. 1992;255(5048):11371141.
3. Larochelle A, Vormoor J, Hanenberg H, et al. Identification of primitive human hematopoietic cells capable of repopulating NOD/SCID mouse bone marrow: implications for gene therapy. Nat Med. 1996;2(12):1329-1337.

4. Ohbo K, Suda T, Hashiyama M, et al. Modulation of hematopoiesis in mice with a truncated mutant of the interleukin-2 receptor gamma chain. Blood. 1996;87(3):956-967.

5. Duarte D, Hawkins ED, Akinduro O, et al. Inhibition of endosteal vascular niche remodeling rescues hematopoietic stem cell loss in AML. Cell Stem Cell. 2018;22(1):64-77.

6. Hawkins ED, Duarte D, Akinduro O, et al. T-cell acute leukaemia exhibits dynamic interactions with bone marrow microenvironments. Nature. 2016;538(7626):518-522.

7. Jing L, Zon LI. Zebrafish as a model for normal and malignant hematopoiesis. Dis Model Mech. 2011;4(4):433-438.

8. Yan C, Yang Q, Do D, Brunson DC, Langenau DM. Adult immune compromised zebrafish for xenograft cell transplantation studies. EBioMedicine. 2019;47:24-26.

9. Corkery DP, Dellaire G, Berman JN. Leukaemia xenotransplantation in zebrafish--chemotherapy response assay in vivo. Br J Haematol. 2011:153(6):786-789.

10. North TE, Goessling W, Walkley CR, et al. Prostaglandin E2 regulates vertebrate haematopoietic stem cell homeostasis. Nature. 2007;447(7147):1007-1011

11. Cutler C, Multani P, Robbins D, et al. Prostaglandin-modulated umbilical cord blood hematopoietic stem cell transplantation. Blood. 2013:122(17):3074-3081.

12. Brocker C, Thompson D, Matsumoto A, Nebert DW, Vasiliou V. Evolutionary divergence and functions of the human interleukin (IL) gene family. Hum Genomics. 2010;5(1):30-55.

13. Wunderlich M, Chou FS, Link KA, et al. AML xenograft efficiency is significantly improved in NOD/SCID-IL2RG mice constitutively expressing human SCF, GM-CSF and IL-3. Leukemia. 2010;24(10): 1785-1788

14. Coughlan AM, Harmon C, Whelan S, et al. Myeloid engraftment in humanized mice: impact of granulocyte-colony stimulating factor treatment and transgenic mouse strain. Stem Cells Dev. 2016;25 (7):530-541.

15. Rajan V, Melong N, Wong WH, et al. Humanized zebrafish enhance human hematopoietic stem cell survival and promote acute myeloid leukemia clonal diversity. Haematologica. 2020;105(10:2391-2399.

16. Bentley VL, Veinotte CJ, Corkery DP, et al. Focused chemical genomics using zebrafish xenotransplantation as a pre-clinical therapeutic platform for T-cell acute lymphoblastic leukemia. Haematologica. 2015;100(1):70-76.

17. Hamilton N, Sabroe I, Renshaw SA. A method for transplantation of human HSCs into zebrafish, to replace humanised murine transplantation models. F1000Res. 2018;7:594.

18. Parada-Kusz M, Penaranda C, Hagedorn EJ, et al. Generation of mouse-zebrafish hematopoietic tissue chimeric embryos for hematopoiesis and host-pathogen interaction studies. Dis Model Mech. 2018;11(11):dmm034876.

19. Tamplin OJ, Durand EM, Carr LA, et al. Hematopoietic stem cell arrival triggers dynamic remodeling of the perivascular niche. Cell. 2015;160(1-2):241-252

20. Koelle SJ, Espinoza DA, Wu C, et al. Quantitative stability of hematopoietic stem and progenitor cell clonal output in rhesus macaques receiving transplants. Blood. 2017;129(11):1448-1457. 